\title{
Influence of correlated impurities on conductivity of graphene sheets: Time- dependent real-space Kubo approach
}

T. M. Radchenko, Artsem Shylau and Igor Zozoulenko

\section{Linköping University Post Print}

N.B.: When citing this work, cite the original article.

Original Publication:

T. M. Radchenko, Artsem Shylau and Igor Zozoulenko, Influence of correlated impurities on conductivity of graphene sheets: Time-dependent real-space Kubo approach, 2012, Physical Review B. Condensed Matter and Materials Physics, (86), 3, 035418.

http://dx.doi.org/10.1103/PhysRevB.86.035418

Copyright: American Physical Society http://www.aps.org/

Postprint available at: Linköping University Electronic Press

http://urn.kb.se/resolve?urn=urn:nbn:se:liu:diva-79666 


\title{
Influence of correlated impurities on conductivity of graphene sheets: Time-dependent real-space Kubo approach
}

\author{
T. M. Radchenko, ${ }^{1,2}$ A. A. Shylau, ${ }^{1}$ and I. V. Zozoulenko, ${ }^{1, *}$ \\ ${ }^{1}$ Solid State Electronics, Department of Science and Technology (ITN), Linköping University, 60174 Norrköping, Sweden \\ ${ }^{2}$ Department of Solid State Theory, Institute for Metal Physics, NASU, 36 Acad. Vernadsky Blvd., 03680 Kyiv, Ukraine
}

(Received 23 May 2012; revised manuscript received 20 June 2012; published 13 July 2012)

\begin{abstract}
Numerical calculations of the conductivity of graphene sheets with random and correlated distributions of disorders have been performed using the time-dependent real-space Kubo formalism. The disorder was modeled by the long-range Gaussian potential describing screened charged impurities and by the short-range potential describing neutral adatoms both in the weak and strong scattering regimes. Our central result is that correlation in the spatial distribution for the strong short-range scatterers and for the long-range Gaussian potential do not lead to any enhancement of the conductivity in comparison to the uncorrelated case. Our results strongly indicate that the temperature enhancement of the conductivity reported in the recent study [J. Yan and M. S. Fuhrer, Phys. Rev. Lett. 107, 206601 (2011)] and attributed to the effect of dopant correlations was most likely caused by other factors not related to the correlations in the scattering potential.
\end{abstract}

DOI: 10.1103/PhysRevB.86.035418

PACS number(s): 72.80.Vp, 72.10.Fk

\section{INTRODUCTION}

Investigation of charge transport in graphene and understanding factors that affect its conductivity represent one of the central directions of graphene research. ${ }^{1-3}$ This is motivated by both fundamental interest to graphene's transport properties as well as by potential applications of this novel material for electronics. It is commonly recognized that the major factors determining the electron mobility in graphene are long-range charged impurities trapped on the substrate and strong resonant short-range scatterers due to adatoms covalently bound to graphene. ${ }^{2}$ The nature of scatterers is directly reflected in the dependence of the conductivity on the electron density $\sigma=$ $\sigma(n)$, and therefore investigation of this function constitutes the focus of experimental and theoretical research. ${ }^{2,3}$ For example, both the strong short-range potential ${ }^{4-9}$ and the long-range potential $^{7,10-12}$ lead to similar linear density dependence of the conductivity commonly observed in experiments. ${ }^{13-19}$ Experiments also often show strong deviations from this linear dependence and an asymmetry with respect to the polarity of carriers, which can be attributed to a number of factors such as the effect of realistic structural defects and impurities, ${ }^{8,20-24}$ effect of contacts, ${ }^{25-27}$ effect of weak shortrange impurities, ${ }^{4,10-12}$ the ballistic or quasiballistic transport regimes, ${ }^{7,28-30}$ ripples, ${ }^{30-32}$ and many others.

Detailed studies of the density dependence of the conductivity in graphene often require exact numerical approaches for transport calculations combined with ab initio calculations for microscopic properties of realistic scatterers. ${ }^{8,20-23}$ Such approaches can capture the essential features of the system at hand as well as can address transport regimes which are not accessible by other means. Numerical approaches can also be used to test a validity of conclusions of various semiclassical analytical approaches and applicability of approximations used in such approaches. Among the most popular numerical methods reported in the literature are the recursive Green's function technique $\mathrm{e}^{33-35}$ and the time-dependent real-space Kubo method. ${ }^{8,9,22-24,36-40}$ The latter method is especially suited to treat large graphene systems containing tens of millions of atoms with dimensions approaching realistic systems.
Recently, Yan and Fuhrer ${ }^{41}$ addressed the effect of correlation in the spatial distribution of disorder on the conductivity of graphene sheet by doping it with potassium atoms. They found that the conductivity of the system at hand increases as the temperature rises, and argued that this was caused by the enhancement of correlation between the potassium ions due to the Coulomb repulsion. This conclusion, in turn, was based on the theoretical predictions of $\mathrm{Li}$ et al. ${ }^{42}$ that the correlations in the position of long-range scatterers strongly enhance the conductivity. It should be noted that the approach of $\mathrm{Li}$ et al ${ }^{42}$ is based on the standard Boltzmann approach within the Born approximation. However, the applicability of the Born approximation for graphene has been questioned in Ref. 7 where it was shown that predictions based on the standard semiclassical Boltzmann approach within the Born approximation for the case of the long-range Gaussian potential are in quantitative and qualitative disagreement with the exact quantum mechanical results in the parameter range corresponding to realistic systems. (A discussion of various aspects of the applicability of the Born approximation for graphene and bilayer graphene can be found in Refs. 7,9, and 43). It is therefore not clear to what extent the semiclassical predictions of $\mathrm{Li}$ et al..$^{42}$ based on the Born approximation are justified for the case of correlated impurities. Since the conclusions of the experiment of Yan and Fuhrer ${ }^{41}$ rely essentially on the above semiclassical predictions, it is of interest to study the effect of spatial correlation of disorders by exact numerical methods.

The main aim of this paper is therefore to investigate the influence of the spatial correlation of disorder on the conductance of graphene sheets using a numerical quantum mechanical approach. In this study, we utilize the timedependent real-space quantum Kubo method, ${ }^{8,9,22-24,36-40}$ allowing us to study large graphene sheets containing millions of atoms. We consider models of disorder appropriate for realistic impurities that might exhibit correlations, including the Gaussian potential describing screened charged impurities and the short-range potential describing neutral adatoms. 


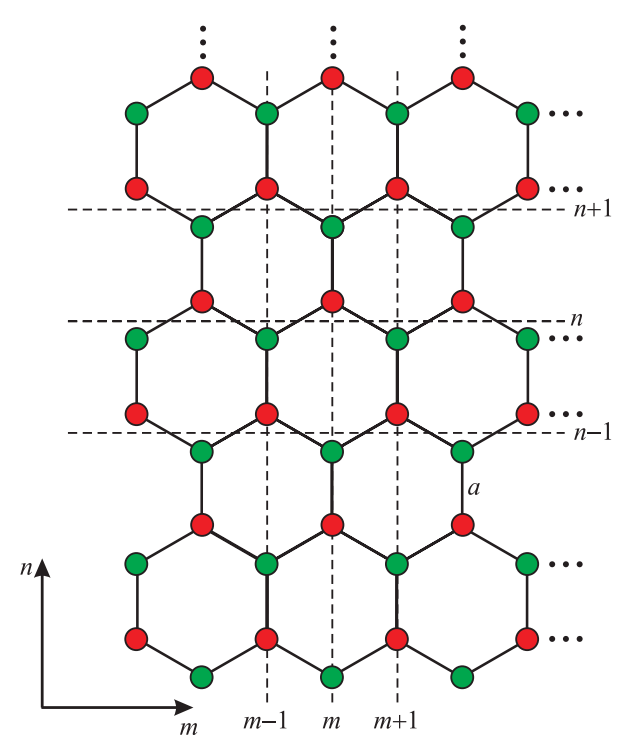

FIG. 1. (Color online) A honeycomb graphene lattice.

The paper is organized as follows. The basic model of the system under consideration including models for impurity potentials as well as the basics of the numerical Kubo method are formulated in Sec. II. Section III presents and discusses the obtained results and compares our numerical findings with available experimental data and theoretical predictions. The conclusions are summarized in Sec. IV. Various details of the numerical approach are presented in the Appendix.

\section{TIGHT-BINDING MODEL AND TIME-DEPENDENT REAL-SPACE KUBO FORMALISM}

We model electron dynamics in graphene using the standard $p$-orbital nearest-neighbor tight-binding Hamiltonian defined on a honeycomb lattice ${ }^{2,3}$ (Fig. 1):

$$
\hat{H}=-u \sum_{i, i^{\prime}} c_{i}^{\dagger} c_{i^{\prime}}+\sum_{i} V_{i} c_{i}^{\dagger} c_{i},
$$

where $c_{i}^{\dagger}$ and $c_{i}$ are the standard creation and annihilation operators acting on a quasiparticle on the site $i=(m, n)$. The summation over $i$ runs over the entire graphene lattice, while $i^{\prime}$ is restricted to the sites next to $i ; u=2.7 \mathrm{eV}$ is the hopping integral for the neighboring atoms $i$ and $i^{\prime}$, and $V_{i}$ is the onsite potential describing impurity scattering.

In this study, we consider both short- and long-range impurities. The short-range impurities represent neutral adatoms on graphene and are modeled by the delta-function potential

$$
V_{i}=\sum_{j=1}^{N_{\text {imp }}} V_{j} \delta_{i j}
$$

where $N_{\text {imp }}$ is the number of impurities on a graphene sheet. Estimations based on ab initio calculations and the $T$-matrix approach for common adatoms provide typical values for the onsite potential $V_{j}=V_{0} \lesssim 80 u .^{9,20,21,35,44}$ (For example, for hydrogen, $V_{0} \approx 60 u$.)

The long-range impurities correspond to charged ions situated typically on a surface of a dielectric substrate. We model them by the Gaussian potential commonly used in the
TABLE I. Relation between the relative concentration of impurities $n_{i}$ and the largest correlation distance $r_{0_{\max }}$ (expressed in units of the carbon-carbon distance $a=0.142 \mathrm{~nm}$ ).

\begin{tabular}{lcccccc}
\hline \hline$n_{i}$ & $0.5 \%$ & $1 \%$ & $2 \%$ & $3 \%$ & $4 \%$ & $5 \%$ \\
\hline$r_{0_{\max }}(a)$ & 13 & 9 & 6 & 5 & 4 & 3 \\
\hline \hline
\end{tabular}

literature: $2,3,7,43,45$

$$
V_{i}=\sum_{j=1}^{N_{\text {imp }}} U_{j} \exp \left(-\frac{\left|\mathbf{r}_{i}-\mathbf{r}_{j}\right|^{2}}{2 \xi^{2}}\right),
$$

where $\mathbf{r}_{i}$ is the radius vector of the site $i, \xi$ is the effective screening length, and the potential height is uniformly distributed in the range $U_{j} \in[-\Delta, \Delta]$ with $\Delta$ being the maximum potential height.

We consider two cases of impurity distribution: random (uncorrelated) and correlated. In the first case of uncorrelated impurities, the summation in Eqs. (2) and (3) is performed over the random distribution of impurities over the honeycomb lattice. In the second case, impurities are no longer considered to be randomly distributed, and to describe their spatial correlation we adopt a model used in Ref. 42 introducing the pair distribution function $g(r)$ :

$$
g(r)= \begin{cases}0, & r<r_{0} \\ 1, & r>r_{0}\end{cases}
$$

where $r$ is the distance between two impurities and the correlation length $r_{0}$ defines the minimum distance that can separate two impurities. [Note that for the randomly distributed (uncorrelated) impurities, $r_{0}=0$.] The largest distance $r_{0_{\max }}$ depends on the relative impurity concentration $n_{i}$; the smaller the concentration $n_{i}$, the larger $r_{0_{\max }}$. Calculated values of $r_{0_{\max }}$ for the different relative impurity concentrations $n_{i}$ are presented in Table I, and representative examples of random and correlated distributions for the cases of the short- and long-range potentials are shown in Fig. 2.

The transport properties of graphene sheets are calculated on the basis of the time-dependent real-space Kubo formalism where the dc conductivity $\sigma$ is extracted from the wavepacket temporal dynamics governed by the time-dependent Schrödinger equation. ${ }^{36-40}$ This is a computationally efficient method scaling with a number of atoms in the system $N$, and thus allowing treating very large graphene sheets containing many millions of $\mathrm{C}$ atoms. ${ }^{8,22-24,40}$

The calculation of the dc conductivity starts from the KuboGreenwood formula ${ }^{46}$

$$
\sigma=\frac{2 \hbar e^{2} \pi}{\Omega} \operatorname{Tr}\left[\hat{v}_{x} \delta(E-\hat{H}) \hat{v}_{x} \delta(E-\hat{H})\right],
$$

where $\hat{v}_{x}$ is the $x$ component of the velocity operator, $E$ is the Fermi energy, $\Omega$ is the area of the graphene sheet, and factor 2 accounts for the spin degeneracy. Let us introduce the mean quadratic spreading of a wave packet along the $x$ direction at the energy $E,\left\langle\Delta \hat{X}^{2}(E, t)\right\rangle=\left\langle(\hat{X}(t)-\hat{X}(0))^{2}\right\rangle$ where $\hat{X}(t)=\hat{U}^{\dagger}(t) \hat{X} \hat{U}(t)$ is the position operator in the Heisenberg representation, and $\hat{U}(t)=e^{-i \hat{H} t / \hbar}$ is the timeevolution operator. The conductivity can then be expressed as 

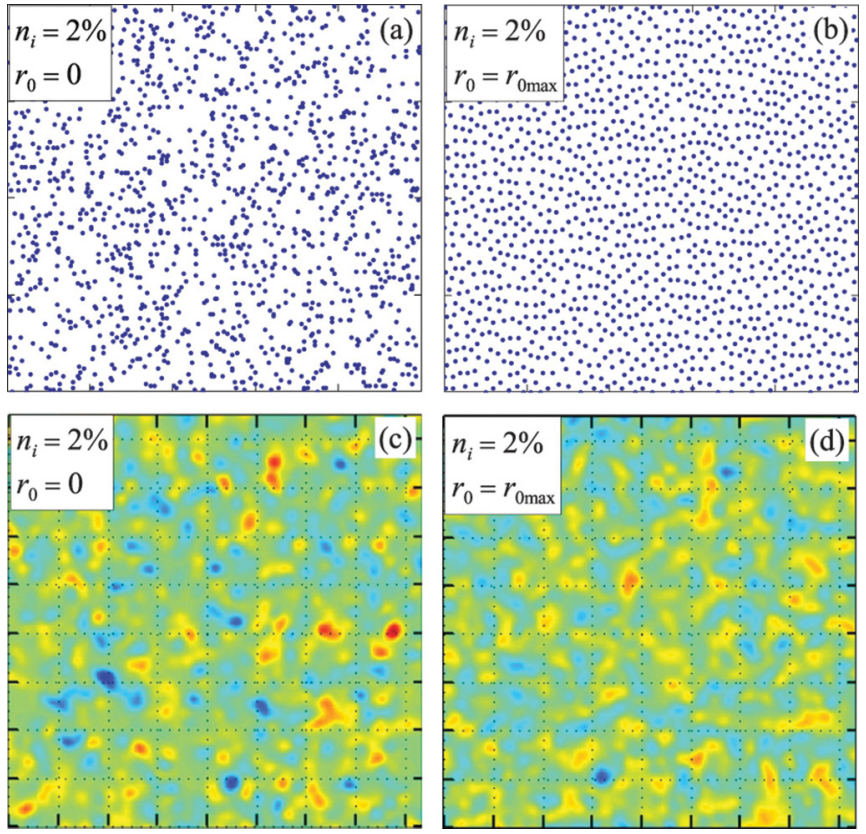

FIG. 2. (Color online) A representative illustration of random and correlated distributions of impurities for the cases of (a), (b) shortrange impurities, and (c), (d) Gaussian impurities for the impurity concentration $n_{i}=2 \%$.

the Einstein relation

$$
\sigma \equiv \sigma_{x x}=e^{2} \tilde{\rho}(E) \lim _{t \rightarrow \infty} D(E, t),
$$

where $\tilde{\rho}(E)=\rho / \Omega=\operatorname{Tr}[\delta(E-\hat{H})] / \Omega$ is the density of sates (DOS) per unit area (per spin), and the time-dependent diffusion coefficient $D(E, t)$ is related to $\Delta \hat{X}^{2}(E, t)($ Ref. 47$)$ :

$$
\begin{aligned}
D(E, t) & =\frac{\left\langle\Delta \hat{X}^{2}(E, t)\right\rangle}{t} \\
& =\frac{1}{t} \frac{\operatorname{Tr}\left[\left(\hat{X}_{H}(t)-\hat{X}(0)\right)^{2} \delta(E-\hat{H})\right]}{\operatorname{Tr}[\delta(E-\hat{H})]} .
\end{aligned}
$$

Hence, calculation of $\sigma$ using the time-dependent real-space Kubo formalism requires numerical evaluation of the mean quadratic spreading of wave packets as prescribed by Eqs. (6) and (7). Details of numerical calculations of $\sigma$ and numerical solution of the time-dependent Schrödinger equation are given in the Appendix.

The diffusion coefficient $D(E, t)$ defined according to Eqs. (7) is generally time dependent and it exhibits different temporal behavior depending on whether the transport regime is (i) ballistic, (ii) diffusive, or (iii) localized. ${ }^{22,23}$ This is illustrated in Fig. 3 showing a temporal evolution of the diffusion coefficient for a graphene sheet with different impurity concentrations $n_{i}$. The corresponding shapes of the wave packets for the different transport regimes are visualized in Fig. 3 for some representative time $t=50 \mathrm{fs}$. In a system with no impurities $\left(n_{i}=0\right)$, electrons propagate ballistically such that the mean spreading of the wave packet is $\sqrt{\left\langle\Delta \hat{X}^{2}(E, t)\right\rangle} \simeq v_{F} t$, where $v_{F}$ is the electron Fermi velocity. As a result, the diffusion coefficient increases linearly with time $D \simeq v_{F}^{2} t$, with the slope being given by $v_{F}^{2}$. The diffusive regime when the diffusion coefficient $D$ reaches saturation

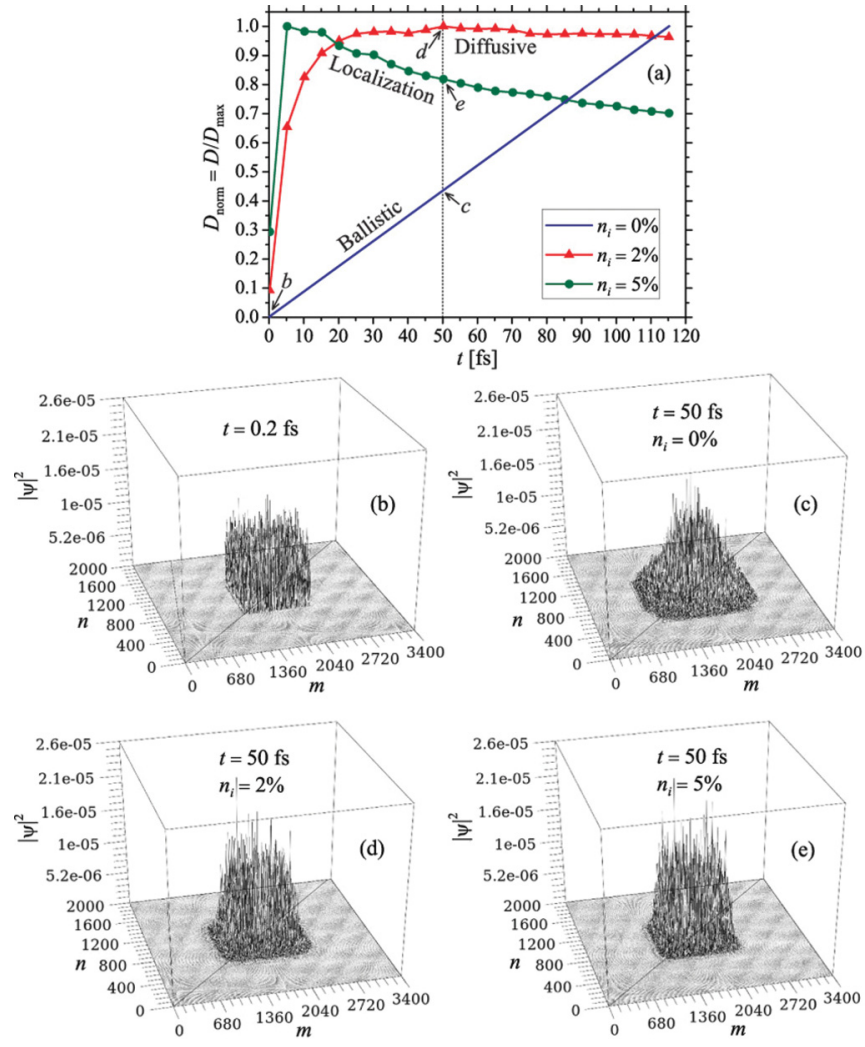

FIG. 3. (Color online) (a) Temporal dependence of the diffusion coefficient for different concentration of strong short-range impurities. The onsite potential $V \sim 37 u$; $E=0.2 u$. (b)-(e) Wave-packet propagation in graphene lattice with different concentration of shortrange impurities. (b) Initial random state of the size $1020 \times 600$ sites given by Eq. (A1). (c)-(e) The wave-packet shape after $t=50 \mathrm{fs}$ for different impurity concentrations corresponding to ballistic, diffusive, and localization regimes from (a).

and becomes independent on time corresponds in Fig. 3 to the impurity concentration $n_{i}=2 \%$. For larger impurity concentration $n_{i}=5 \%$, the system is in the localization regime when the diffusion coefficient decreases with time due to quantum effects leading to the weak or the strong localization. It should be stressed that in this study, we are interested in the diffusive transport regime when the diffusion coefficient reaches its maximum. Therefore, following Refs. 22 and 23, we replace in Eq. (6) $\lim _{t \rightarrow \infty} D(E, t) \rightarrow D_{\max }(E)$, such that the dc conductivity is defined as

$$
\sigma=e^{2} \tilde{\rho}(E) D_{\max }(E) .
$$

It is noteworthy that within the Boltzmann approach the conductivity is given by $\sigma_{\text {Boltz }}=e^{2} \tilde{\rho}(E) \tau \frac{v_{F}^{2}}{2}$, where $\tau$ is the scattering time. Hence, it follows from Eq. (8) that the elastic length is related to the computed diffusion coefficient via $l_{e}=v_{F} \tau=2 D_{\max } / v_{F}$.

In most experiments, the conductivity is measured as a function of the electron density $n$. Having calculated the DOS $\tilde{\rho}(E)$ as described in the Appendix, we compute the electron density $n(E)=\int_{-\infty}^{E} \tilde{\rho}(E) d E-n_{\text {ions }}$, where $n_{\text {ions }}=3.9 \times 10^{15} \mathrm{~cm}^{-2}$ is the density of the positive ions in the graphene lattice compensating the negative charge of the $p$ electrons [note that for the ideal graphene lattice at the neutrality point $n(E)=0]$. 

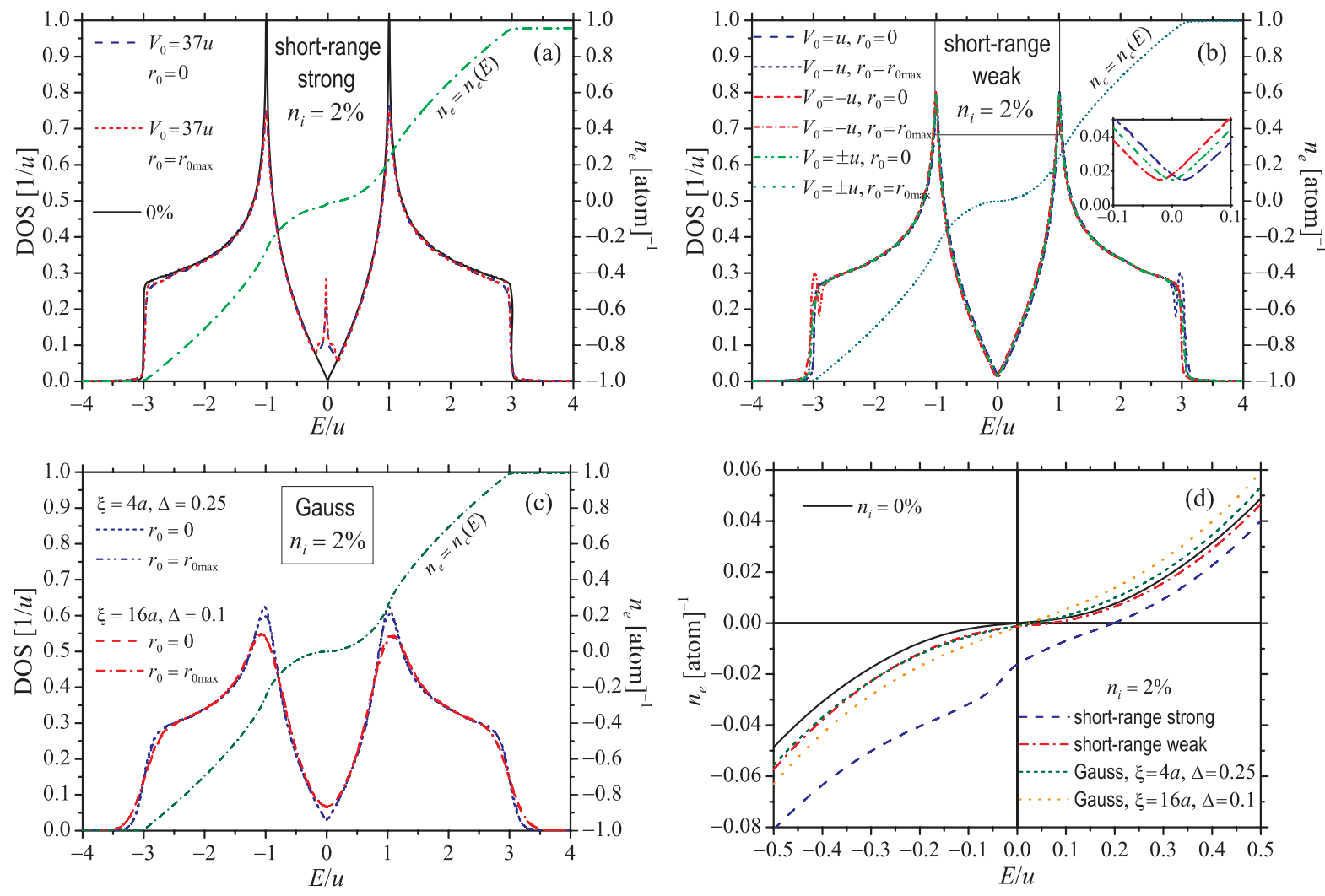

FIG. 4. (Color online) Density of states (DOS) and the relative charge carrier concentration $n_{e}$ (the number of electrons per C atom) as a function of the energy $E$ for (a) short-range strong, (b) short-range weak, and (c) Gaussian potential for random and correlated impurities. (c) Dependencies $n=n(E)$ for short- and long-range potentials.

[A dependence $n=n(E)$ is illustrated in Fig. 4 for different scattering potentials.] Combining the calculated $n(E)$ with the conductivity $\sigma(E)$ given by Eq. (8), we compute the density dependence of the conductivity $\sigma=\sigma(n)$.

\section{RESULTS AND DISCUSSION}

In this section, we present results for the numerical conductivity of the graphene sheets with random and correlated impurities described by the short- and long-range potentials [Eqs. (2) and (3), respectively].

\section{A. Short-range impurities}

Depending on the impurity strength, the conductivity of graphene sheets is expected to exhibit qualitatively different density dependence for the weak and strong short-range scattering. The standard Boltzmann approach within the Born approximation predicts that the conductivity is independent on the electron density ${ }^{2-4,9}$

$$
\sigma=\frac{8 e^{2}}{h} \frac{\left(v_{F} \hbar\right)^{2}}{n_{i} V_{0}^{2}}
$$

where $v_{F}$ is the Fermi velocity in graphene, $v_{F} \hbar=\frac{3}{2} u a$, and $V_{0}$ is the strength of the onsite potential in Eq. (2), $V_{j}=V_{0}$. Going beyond the Born approximation, one obtains that strong scatterers, with the accuracy up to logarithmic corrections, lead to a linear density dependency ${ }^{4-6}$

$$
\sigma=\frac{4 e^{2}}{h} \frac{n}{n_{i}}\left(\ln \sqrt{\pi n} R_{0}\right)^{2},
$$

where $R_{0}$ is the scatterer's radius. Note that the condition for the validity of the Born approximation in Eq. (9) can be presented in the form ${ }^{9,43}\left(\left|V_{0}\right| / u\right)^{-1} \ll \sqrt{n_{e}} \ln n_{e}$, where $n_{e}$ is the relative electron density (i.e., the electron density per $\mathrm{C}$ atom). For realistic electron densities $n_{e} \lesssim 0.01$, this condition loosely corresponds to $\left|V_{0}\right| \lesssim u$, which we adopt as a definition of the weak impurity scattering. The condition $\left|V_{0}\right| \gg u$ [when Eq. (10) is expected to be valid] defines the case of the strong impurity scattering. Note that most of the adatoms present in graphene fall into the second case of the strong scattering with the effective onsite potentials in the range $\left|V_{0}\right| / u \approx 10-80 .{ }^{44}$

\section{Short-range potential, strong scattering $\left|V_{0}\right| \gg u$}

Figures 4(a) and 4(d) show the DOS and the electron density $n=n(E)$ in a graphene sheet with strong scatterers $V_{0}=37 u$ and the concentration $n_{i}=2 \%$. The calculated dependencies are practically identical for the cases of random and correlated distributions of impurities. The calculated DOS shows a pronounced peak in the vicinity of the Dirac point $-0.3 \lesssim \frac{E}{u} \lesssim 0.1$, which reflects a formation of the impurity band as discussed in Ref. 8 . Note that the peak is shifted with respect to $E=0$, which is related to the asymmetry of the impurity potential $\left(V_{0}>0\right)$. The shift of the impurity band 

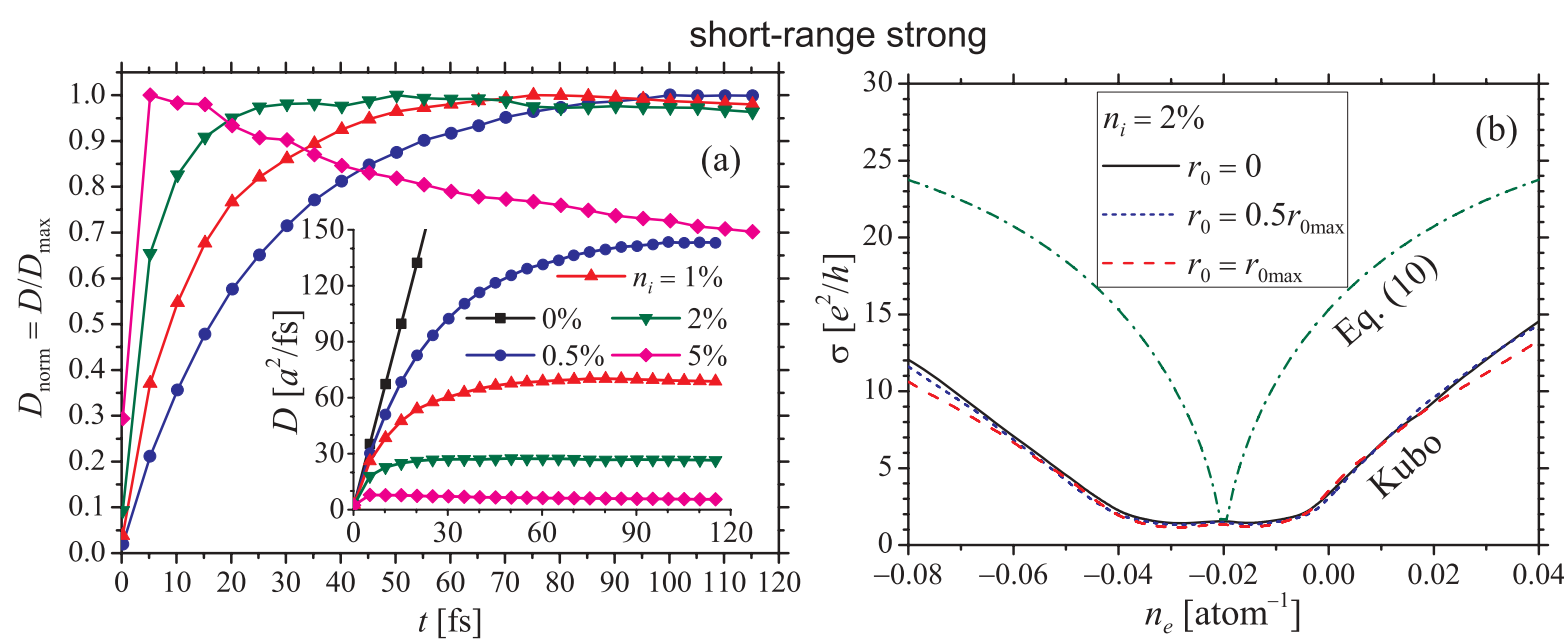

FIG. 5. (Color online) (a) Diffusion coefficient $D=D(t)$ for different concentration of the short-range strong impurities; $V_{0}=37 u$, $E=0.2 u$. (b) The conductivity $\sigma$ as a function of the relative electron density $n_{e}$ (the number of electrons per $\mathrm{C}$ atom) for random and correlated impurities $n_{i}=2 \%$. The dotted-dashed line corresponds to Eq. (10), which is shifted to the charge neutrality point at $n_{e} \approx 0.02$.

also leads to the asymmetry in $n=n(E)$ dependence [see Fig. 4(d)].

Figure 5(a) shows the time-dependent diffusion coefficient for different impurity concentrations for the case of random distribution of impurities. The temporal dependencies $D=$ $D(t)$ demonstrate that the system at hand reaches and stays in the diffusive transport regime for the impurity concentration $n_{i}=1 \%$ and $2 \%$ at times $t \gtrsim 70$ and 30 fs correspondingly. For the impurity concentration $n_{i}=0.5 \%$, the diffusive regime is reached at times outside those shown in the figure $(t \gtrsim$ $130 \mathrm{fs}$ ), whereas for $n_{i}=5 \%$ the system almost immediately reaches the localization regime.

To analyze the density dependence of the conductivity, we choose a representative concentration $n_{i}=2 \%$ and $t=80 \mathrm{fs}$ when $D(t)$ exhibits a saturation corresponding to a well-defined diffusive regime [see Fig. 5(b)]. The randomly distributed impurities show a quasilinear density dependence of the conductivity. It is worth to note that the calculated conductivity fully reproduces numerical results reported by Yuan et $a l^{8}{ }^{8}$ who used a similar time-dependent Kubo approach. The corresponding theoretical prediction exhibits pronounced deviations from the linear dependence caused by logarithmic corrections in Eq. (10). These deviations are much stronger than those typically seen experimentally. ${ }^{2}$ This is related to the fact that in realistic experimental samples, the electron densities are lower than those used in our calculations, ${ }^{48}$ such that at smaller densities Eq. (10) also exhibits a quasilinear dependence. In the vicinity of the Dirac point, the conductivity flattens out, which is related to a transport regime due to a formation of the impurity band. Apparently, the theoretical prediction Eq. (10) does not reproduce this transport regime. For larger densities away from the Dirac point, the calculated Kubo conductivity differs by a factor of $\sim 2$ from the theoretical predictions given by Eq. (10). Note that the calculated dependence $\sigma=\sigma(n)$ is shifted with respect to $n=0$, which is caused by the asymmetry in the dependence $n=n(E)$ due to the impurity band as discussed above.

Figure 5(b) also shows the dependence $\sigma=\sigma(n)$ for the case of correlated impurities with the correlation lengths $r_{0}=0.5 r_{0 \max }$ and $r_{0 \max }$. The central result is that correlation in the impurity distribution practically does not affect the conductivity of the system even for the largest correlation length $r_{0 \text { max }}$.

\section{Short-range potential, weak scattering $\left|V_{0}\right| \lesssim u$}

Let us now turn to the case of weak scattering. We consider two cases: the symmetric potential $V_{0}= \pm u$, and the asymmetric one $V_{0}=u$. Figures 4(b) and 4(d) show the DOS and the electron density $n=n(E)$ for the case of $n_{i}=2 \%$. As in the case of the strong scatterers, the calculated dependencies are practically identical for random and correlated distributions of impurities. However, in contrast to the case of the strong scatterers, the DOS does not form the impurity band close to the Dirac point.

Figure 6 shows the time-dependent diffusion coefficients $D(t)$ and the density dependence of the conductivity for $n_{i}=3 \%$ with random and correlated distributions of disorders. In contrast to the case of the strong scattering potential, in the present case the diffusion coefficient reaches its maximum at the same time $t \approx 120$ fs independent of the impurity concentration. The density dependence of the conductivity $\sigma=\sigma(n)$ for symmetric scatterers is shown in Fig. 6(b). In agreement with the Boltzmann predictions [Eq. (9)], the calculated Kubo conductivity is independent of the carrier density, even though its calculated value differs by a factor of $\sim 3$ from the corresponding theoretical predictions. The calculated $\sigma$ is independent on $n$ for all densities except those in the vicinity of the Dirac point, where the conductivity shows a pronounced dip. This can be explained by the fact that close to the Dirac point, the graphene sheet is in the electron-hole puddle density regime with strong potential variations comparable to the Fermi energy of electrons. Because Eq. (9) is not valid in such a regime, the calculated density strongly deviates from the predictions of Eq. (9). Figure 6(b) also shows the the conductivity for the case of correlated impurities with $r_{0}=0.6 r_{0 \max }$ and $r_{0 \max }$. Similarly to the case of strong scatterers, the correlation in the impurity 

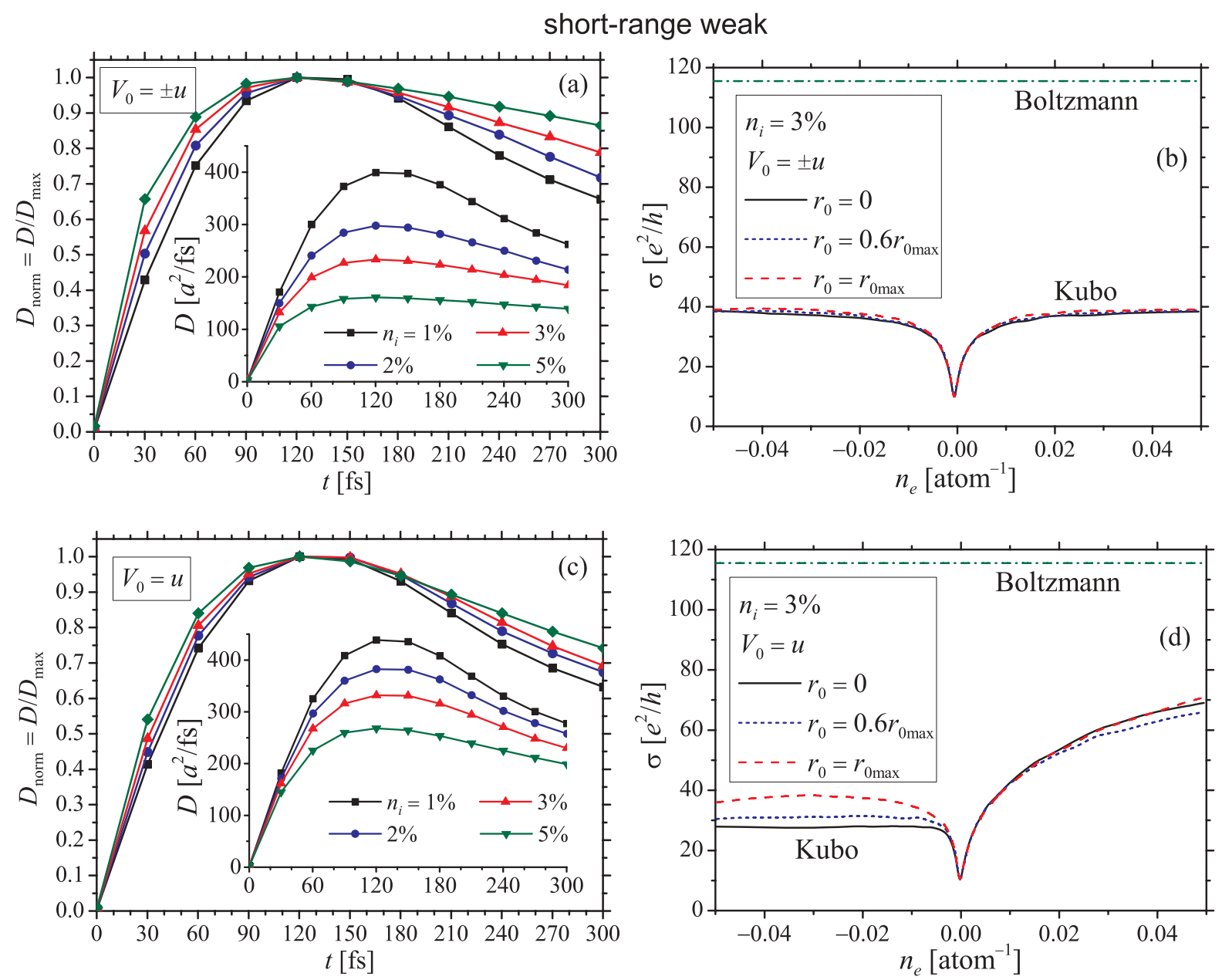

FIG. 6. (Color online) (a), (c) The diffusion coefficient $D=D(t)$ for short-range weak impurities with different concentrations $n_{i}$; $E=0.2 u$. (b), (d) The conductivity $\sigma$ as functions of the relative electron density $n_{e}$ (the number of electrons per $\mathrm{C}$ atom) for random and correlated short-range weak impurities $n_{i}=3 \%$. Dotted-dashed lines show the Boltzmann predictions according to Eq. (9). Panels (a), (b) correspond to the symmetric potential $V_{0}= \pm u$, and (b), (d) for asymmetric one $V_{0}=u$.

distribution practically does not affect the conductivity of the system at hand.

The density dependence of the conductivity $\sigma=\sigma(n)$ for the case of asymmetric potential $V_{0}=u$ is presented in Fig. 6(d). It has two features that are different from the case of the symmetric potential. First, for negative electron densities, the calculated conductivity is independent of $n$, which is consistent with the Boltzmann predictions [Eq. (9)]. However, for positive densities, the conductivity shows a sublinear density dependence distinct from Eq. (9). Second, for the case of correlated impurities, the conductivity is increased by up to $30 \%$ in the region of negative densities (when $\sigma \approx$ const), whereas $\sigma$ is not affected by the correlations for positive densities where the behavior is sublinear. It should be stressed that the Boltzmann theory predicts the same density dependence regardless whether the potential $V_{0}$ is symmetric or not, whereas our numerical calculations show a clear difference between these two cases. Note that calculations with the asymmetric potential $V_{0}=-u$ show the density dependence of the conductivity which is mirror symmetric to the case of $V_{0}=u$.

\section{B. Long-range Gaussian impurities}

Let us now turn to the case of the long-range Gaussian potential [Eq. (3)]. The graphene conductivity calculated within the the standard Boltzmann approach in the Born approximation reads as ${ }^{7,45,49}$

$$
\sigma=\frac{4 e^{2}}{h} \frac{\pi n \xi^{2} e^{\pi n \xi^{2}}}{K I_{1}\left(\pi n \xi^{2}\right)} \propto \begin{cases}\text { const, } & |z| \ll 1 \\ n^{3 / 2}, & |z| \gg 1\end{cases}
$$

where $z \equiv \pi n \xi^{2}=\left(\frac{2 \pi \xi}{\lambda}\right), I_{1}$ is the modified Bessel function, and $K \approx 40.5 n_{i}(\Delta / u)^{2}(\xi / \sqrt{3} a)^{4}$ with $a$ being the carboncarbon distance and $\lambda$ being the Fermi wavelength. The condition $|z| \ll 1$ describes the case of quantum scattering when the Fermi wavelength is larger than the effective screening length $\lambda \gg \xi$, while the opposite condition $|z| \gg 1$ corresponds to the case of classical scattering $\lambda \ll \xi$. Because the semiclassical approach predicts two distinct regimes of conductivity, in our numerical calculations we explore the parameter space corresponding to these two regimes. We consider $\xi=4 a$ and $16 a$, which in the considered density interval $\left|n_{e}\right| \lesssim 0.06$ correspond respectively to $|z| \lesssim 2$ and 

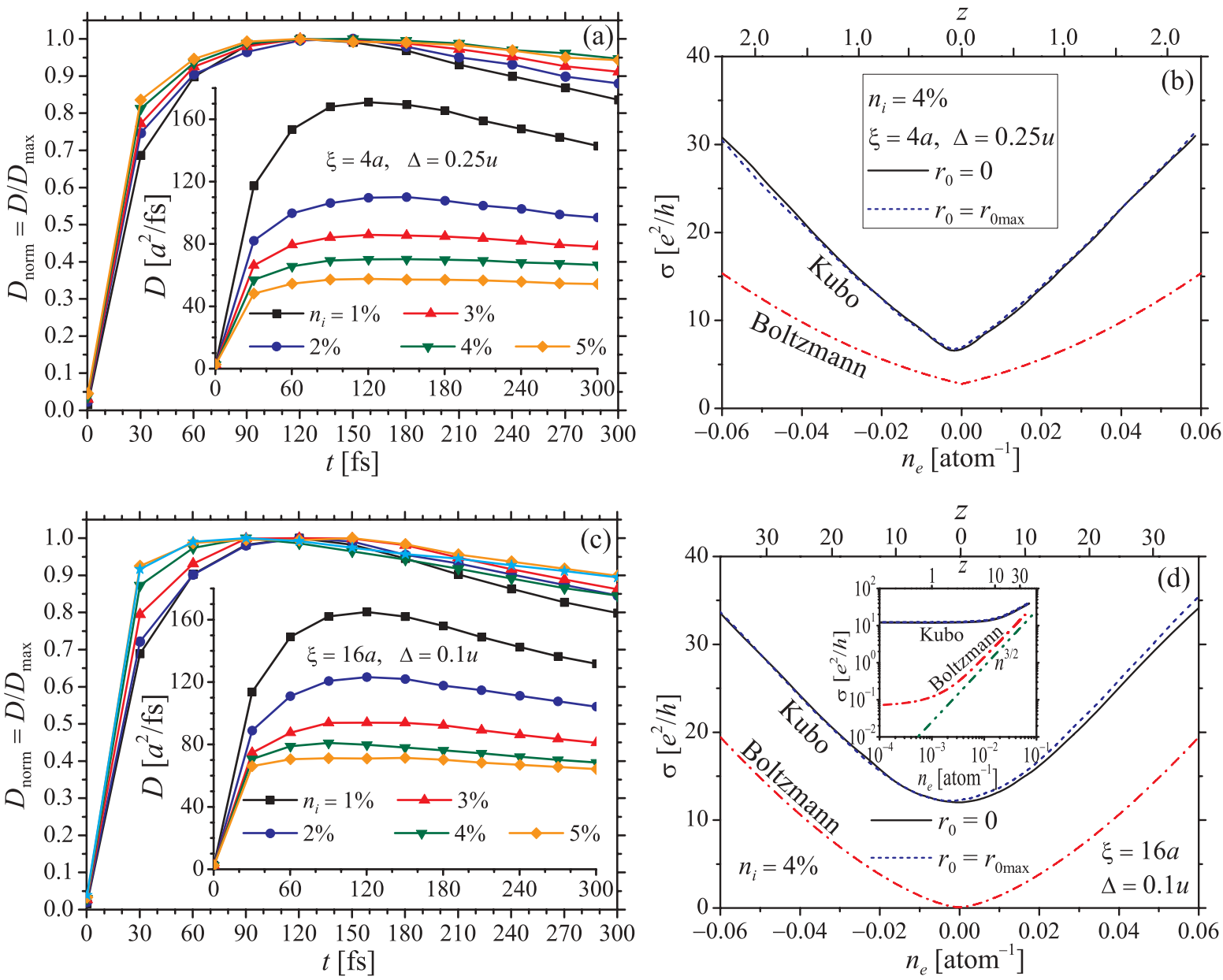

FIG. 7. (Color online) (a), (c) The diffusion coefficient $D=D(t)$ for the Gaussian impurities with different concentrations $n_{i} ; E=0.2 u$. (b), (d) The conductivity $\sigma$ as functions of the relative electron density $n_{e}$ (the number of electrons per $\mathrm{C}$ atom) for random and correlated Gaussian impurities $n_{i}=4 \%$. A dotted-dashed line shows the Boltzmann predictions according to Eq. (11). Inset in (d) is plotted in the logarithmic scale. Panels (a), (b) and (c), (d) correspond to $\xi=4 a$ and $16 a$, respectively.

$|z| \lesssim 35$. Note that the regime $|z| \lesssim 1$ is appropriate to realistic experimental samples, whereas the carrier density $|z| \gtrsim 1$ is too high to be achieved in the experiment. ${ }^{7,43}$

Figures 4(c) and 4(d) show the DOS and the electron density $n=n(E)$ for the case of $n_{i}=2 \%$. The Gaussian potential significantly smooths the density of states especially in the region of the van Hove singularities. As in the case of the shortrange scatterers, the calculated dependencies are practically identical for random and correlated distributions of impurities.

Figure 7 shows the time-dependent diffusion coefficients $D(t)$ and the conductivity $\sigma=\sigma(n)$ for $n_{i}=4 \%$ with random and correlated distributions of disorders. As in the case of the weak short-range disorder in the present case, the diffusion coefficient reaches its maximum value $D_{\max }$ at the same time for all impurity concentrations studied $1 \% \leqslant n_{i} \leqslant 5 \%(t=$ 130 fs respectively 110 fs for $\xi=4 a$ respectively $16 a$ ).

The density dependence of the conductivity for $\xi=4 a$ is shown in Fig. 7(b) in the density interval $|z| \lesssim 2$. The conductivity exhibits the linear density dependence which well extends into the classical regime $|z|>1$. This is in agreement with previous numerical calculations performed using the Green's function technique, ${ }^{7,33}$ also showing linear (or quasilinear) density dependence of the conductivity. On the contrary, the standard Boltzmann approach predicts that for $|z| \ll 1$ the conductivity is independent on the electron density $\sigma=$ const. For the present case of the Gaussian potential, it has been argued that the standard Boltzmann approach (leading to $\sigma=$ const) is not valid because the Born approximation is not well justified. ${ }^{7}$

Figure 7(d) shows the conductivity for the case of Gaussian disorder for $\xi=16 a$ in the density interval $|z| \lesssim 35$. Even in this case, the calculated conductivity shows the linear density dependence $\sigma \propto n$. For $|z| \gg 1$, the Boltzmann approach predicts the superlinear dependence $\sigma \propto n^{3 / 2}$ [this is clearly discernible in the logarithmic scale as shown in the inset of Fig. 7(d)]. We, however, do not reach such high densities to reproduce this asymptote. (This regime of the high densities was analyzed in Ref. 45.)

Figures 7(b) and 7(d) also show the conductivity for the case of the correlated impurity distribution for $\xi=4 a$ and $16 a$. Even for the maximal correlation between impurities $r_{0}=r_{0 \max }$, the correlation practically does not affect the conductivity. This represents the central result of this section. 


\section{Comparison to the experiment}

Let us now compare our numerical data with available experimental results and theoretical predictions. Recently, Yan and Fuhrer reported conductivity measurements of potassiumdoped graphene. ${ }^{41}$ They found that with the increase of the temperature, the conductivity of doped graphene increases by up to a factor of 2 . They attributed this effect to the enhancement of the spatial correlation between potassium ions due to the mutual Coulomb repulsion, which, according to the recent theory of $\mathrm{Li}$ et al. ${ }^{42}$ leads to the conductivity enhancement. Our numerical calculations, however, do not support this conclusion. Indeed, we demonstrated that the spatial correlations of charged impurities (modeled by the long-range Gaussian potential) have no effect on the conductivity of graphene sheets. Our results therefore suggest that the enhancement of the conductivity with increase of the temperature in experiments of Yan and Fuhrer ${ }^{41}$ is most likely caused by other factors not related to the correlations of impurities. Moreover, as no direct evidence of the spatial correlation of the potassium ions was presented in the above article, it is not clear whether impurities in experimental samples are correlated at the first place, and whether this correlation (if any) increases with the temperature.

Our numerical results do not apparently support theoretical predictions of Li et al. ${ }^{42}$ that the correlations in the impurity positions lead to the enhancement of the conductivity. As mentioned before, it has been shown that for the long-range Gaussian potential in a parameter range corresponding to realistic systems, the standard Boltzmann predictions are in quantitative and qualitative disagreement with the numerical results. ${ }^{7}$ The reason for that is the utilization of the Born approximation, which relies on the unperturbed wave functions of ideal graphene without impurities. This is justified only for the case of weak scattering and apparently fails for the long-range Gaussian scatterers in the parameter range corresponding to realistic systems. (For the discussion of the Born approximation for graphene and bilayer graphene, see Refs. 7,9 and 43.) This explains the discrepancy between the numerical results and predictions of $\mathrm{Li}$ et al., ${ }^{42}$ which are essentially based on the standard Boltzmann approach in the Born approximation.

\section{CONCLUSIONS}

Using an efficient time-dependent real-space Kubo formalism, we performed numerical studies of conductivity of large graphene sheets with random and correlated distribution of disorder. In order to describe realistic disorder, we used models of the short-range scattering potential (appropriate for adatoms covalently bound to graphene) and the long-range Gaussian potential (appropriate for screened charged impurities on graphene and/or dielectric surface). The calculations for the uncorrelated potentials are compared to the corresponding predictions based on the semiclassical Boltzmann approach and to exact numerical calculations performed by different methods.

We find that for the most important experimentally relevant cases of disorder, namely, the strong short-range potential and the long-range Gaussian potential, the correlation in the distribution of disorder does not affect the conductivity of the graphene sheets as compared to the case when disorder is distributed randomly. This represents the main result of our study. We find that the correlations lead to the enhancement of the conductivity only for the case of the weak short-range potential and only when the potential is asymmetric, i.e., $V=V_{0}$ or $V=-V_{0}$. No enhancement of the conductivity is found for the symmetric weak short-range potential $V= \pm V_{0}$.

Using our results, we analyze the recent experiment of Yan and Fuhrer ${ }^{41}$ where the temperature increase of the conductivity was attributed to the enhancement in the spatial correlation of the adsorbed potassium ions. Our numerical findings do not sustain this interpretation and our results strongly suggest that the enhancement of the conductivity reported in the above study is most likely caused by other factors not related to the correlations of impurities.

Our numerical calculations do not support theoretical predictions of $\mathrm{Li}$ et $a l .{ }^{42}$ that the correlations in the impurity positions for the long-range potential lead to the enhancement of the conductivity. We attribute this to the utilization of the standard Boltzmann approach within the Born approximation, which is not justified for the case of a long-range potential in the parameter range corresponding to realistic systems.

\section{ACKNOWLEDGMENTS}

The authors greatly appreciate discussions with A.-P. Jauho and $\mathrm{M}$. Brandbyge concerning the time-dependent Kubo formalism. A financial support from the Swedish Institute is greatly acknowledged.

\section{APPENDIX : NUMERICAL CALCULATION OF THE dc CONDUCTIVITY $\sigma$ ON THE BASIS OF THE TIME-DEPENDENT REAL-SPACE KUBO METHOD}

\section{Calculation of the DOS $\rho(E)$ and the time-dependent diffusion coefficient $D(E, t)$}

Numerical calculation of the dc conductivity $\sigma$ requires computation of the $\operatorname{DOS} \rho(E)=\operatorname{Tr}[\delta(E-\hat{H})]$ and the timedependent diffusion coefficient $D(E, t)$ [Eqs. (7)]. Let us start with an algorithm for calculation of $\rho(E)$.

Express the density of states $\rho(E)$ as a sum over the local densities of states $\rho(E)=\sum_{i}^{N} \rho_{i}(E)$, where the summation is performed over all the sites of graphene lattice $N$. In its turn, the local density of states is given by the imaginary part of the diagonal elements of the Green's function $\rho_{i}(E)=$ $-\frac{1}{\pi} \operatorname{Im} G_{i i}(E+i \varepsilon)$, where a small $\varepsilon \rightarrow 0$ is introduced in order to smooth the peaks in the DOS.

There is an efficient numerical algorithm for calculation of the diagonal elements $G_{i i}$. It first starts with the tridiagonalization procedure when the Hamiltonian is reduced to the tridiagonalized form by passing to a new basis. Then, the first diagonal element of the Green's function $G_{11}$ is computed using the standard continued fraction technique. The details of this algorithm are presented in this Appendix. Note that this algorithm scales as the number of sites $N$, with the most time-consuming part being the tridiagonalization procedure. Having calculated the local density of states for the first site 
$\rho_{1}(E)$, one can in principle repeat similar calculations for all remaining $N-1$ sites in order to recover the total DOS $\rho(E)$. This would apparently require total computation efforts that scale as $N^{2}$. For disordered systems treated in this paper, one can adopt a different way to evaluate $\rho(E)$ that still keeps the total computation efforts of the order of $N$. It relies on the fact that a sufficiently large subsystem of the total system has the same density of states as the original one. Our computation of $\rho(E)$ proceeds then as follows.

Choose a subsystem occupying $M$ sites of the graphene lattice. Construct a random state $\left|\psi_{r}\right\rangle$ extended over these $M$ sites,

$$
\left|\psi_{\mathrm{ran}}\right\rangle=\frac{1}{\sqrt{M}} \sum_{i} e^{2 i \pi \alpha_{i}}|i\rangle
$$

with $\alpha_{i}$ being the random phase in the interval $[0,1],|i\rangle=$ $c_{i}^{\dagger}|0\rangle$, and the summation is extended over the sites of the chosen subsystem. An example of such a random state is shown in Fig. 3(b). Transform the original tight-binding Hamiltonian (1) defined in the basis $\left\{r_{i}\right\}$ by passing on to a new basis as follows. Choose the first element of the new basis as $\mid 1\}=\left|\psi_{\text {ran }}\right\rangle$, where $\left|\psi_{\text {ran }}\right\rangle$ is the random state defined according to Eq. (A1). Tridiagonalize the Hamiltonian as prescribed in this Appendix and calculate $\rho_{1}(E)=-\frac{1}{\pi} \operatorname{Im} G_{11}(E+i \varepsilon)$ using the continued fraction technique. Repeat this procedure, if needed, for different distributions of disorder and average $\rho_{1}(E)$ over these disorder realizations. The calculated value of $\rho_{1}(E)$ corresponds to the DOS per carbon atom of the system at hand. Note that calculation of the remaining $N-1$ matrix elements $G_{i i}$ is not needed such that the total computational efforts still scale as $N$.

Calculation of the trace $\operatorname{Tr}\left[\left(\hat{X}_{H}(t)-\hat{X}(0)\right)^{2} \delta(E-\hat{H})\right]$ in the expression for $D(E, t)$ in Eqs. (7) is performed in a similar way. It can be shown ${ }^{39}$ that this trace can be represented as a sum of the local densities of states $\operatorname{Tr}\left[\left(\hat{X}_{H}(t)-\hat{X}(0)\right)^{2} \delta(E-\right.$ $\hat{H})]=\sum_{i}^{N} \rho_{i}(E, t)$ for the functions $\Psi_{i}(t)$, where

$$
\rho_{i}(E, t)=\sum_{i=1}^{N}\left\langle\Psi_{i}(t)|\delta(E-\hat{H})| \Psi_{i}(t)\right\rangle
$$

and

$$
\left|\Psi_{i}(t)\right\rangle=\hat{X} \hat{U}\left|\psi_{i}\right\rangle-\hat{U}\left(\hat{X}\left|\psi_{i}\right\rangle\right)
$$

where $\hat{X}$ is the position operator (i.e., the $x$ coordinate), $\hat{U}$ is the time-evolution operator, and $\left\{\psi_{i}\right\}$ is the orthogonal basis set [corresponding to, e.g., the eigenstates of the Hamiltonian (1)]. Next, we pass on to the new basis setting its first element $\mid 1\}=\left|\Psi_{i}(t)\right\rangle$, where $\left|\Psi_{i}(t)\right\rangle$ is given by Eq. (A3) where $\left|\psi_{i}\right\rangle=$ $\left|\psi_{\text {ran }}\right\rangle$ is chosen as the random state defined by Eq. (A1). Time evolution of the random initial state in Eq. (A3) is calculated by means of the Chebyshev method as described below. We then reduce the Hamiltonian to the tridiagonalized form and use the continued fraction technique to calculate local density of states $\rho_{1}(E, t)$ in the new bases. As argued above, for a sufficiently large random state, this local density of state well approximates the density of states of the whole system.

\section{Exact solution of the time-dependent Schrödinger equation by means of the Chebyshev method}

In this Appendix, we present an efficient method for solution of the time-dependent Schrödinger equation based on the expansion of the time-evolution operator in an orthogonal set of Chebyshev polynomials.

We start from the time-dependent Schrödinger equation complemented by the initial condition

$$
\hat{H}|\psi(t)\rangle=i \hbar \frac{\partial}{\partial t}|\psi(t)\rangle, \quad|\psi(t=0)\rangle=\left|\psi_{0}\right\rangle,
$$

with $\hat{H}$ being the tight-binding time-independent Hamiltonian (1). Its formal solution can be expressed via the timeevolution operator $\hat{U}(t)$ :

$$
|\psi(t)\rangle=\hat{U}(t)\left|\psi_{0}\right\rangle, \quad \hat{U}(t)=e^{-\frac{i}{\hbar} \hat{H}(t)}
$$

In order to expand $\hat{U}(t)$ in a set of the Chebyshev polynomials $T_{n}(x)$ (which are defined in the interval $x \in[-1 ; 1]$ ), we first renormalize the Hamiltonian such that its spectrum lies in the above interval,

$$
\hat{H}_{\mathrm{norm}}=\frac{2 \hat{H}-\left(E_{\max }+E_{\min }\right) \hat{I}}{E_{\max }-E_{\min }},
$$

where $E_{\max }$ and $E_{\min }$ are the largest and the smallest eigenvalues of the original Hamiltonian (1). (In order to calculate $E_{\max }$ and $E_{\min }$, we use a computational routine that estimates the largest/smallest eigenvalues of the operator without calculation of all the eigenvalues.)

By expanding $\hat{U}(t)$ in Chebyshev polynomials in Eq. (A5), we obtain for the wave function

$$
|\psi(t)\rangle=\sum_{n=0}^{\infty} c_{n}(t)\left|\Phi_{n}\right\rangle
$$

where the functions $\left|\Phi_{n}\right\rangle=T_{n}\left(\hat{H}_{\text {norm }}\right)\left|\psi_{0}\right\rangle$ are calculated using the recurrence relations for the Chebyshev polynomials

$$
\left|\Phi_{n+1}\right\rangle=2 \hat{H}_{\mathrm{norm}}\left|\Phi_{n}\right\rangle-\left|\Phi_{n-1}\right\rangle
$$

with $\left|\Phi_{0}\right\rangle=T_{0}\left(\hat{H}_{\text {norm }}\right)\left|\psi_{0}\right\rangle=\left|\psi_{0}\right\rangle$. The expansion coefficients $c_{n}(t)$ are calculated making use of the orthogonality relation for the Chebyshev polynomials:

$$
c_{n}(t)=2 e^{-i \frac{\left(E_{\max }+E_{\min }\right) t}{2 \hbar}}(-i)^{n} J_{n}\left(\frac{E_{\max }-E_{\min }}{2 \hbar} t\right) .
$$

For large $t$, the expansion coefficients $c_{n}(t)$ become exponentially small. This leads to the fast convergence of the expansion series Eq. (A7), and makes the Chebyshev method very efficient for calculation of the temporal dynamics. For instance, in our simulations, we take 10000 iterations for $300 \mathrm{fs}$ of elapsed time. 


\section{Continued fraction technique and tridiagonalization of the Hamiltonian matrix \\ a. Continued fraction technique}

Consider a Hamiltonian matrix in a tridiagonal form

$$
\hat{H}_{\mathrm{tri}}=\left(\begin{array}{ccccccc}
\alpha_{1} & \beta_{1} & 0 & \ldots & \ldots & \ldots & \ldots \\
\beta_{1} & \alpha_{2} & \beta_{2} & 0 & \ldots & \ldots & \ldots \\
0 & \beta_{2} & \alpha_{3} & \beta_{3} & 0 & \ldots & \ldots \\
\vdots & 0 & \beta_{3} & \ddots & \ddots & \ddots & \ldots \\
\vdots & \vdots & 0 & \ddots & \ddots & \ddots & \ddots \\
\vdots & \vdots & \vdots & \ddots & \ddots & \ddots & \beta_{N-1} \\
\vdots & \vdots & \vdots & \vdots & \ddots & \beta_{N-1} & \alpha_{N}
\end{array}\right) .
$$

The continued fraction technique provides an efficient way to calculate the first diagonal element $G_{11}$ of the Green's function $\hat{G}=\left(E \hat{I}-\hat{H}_{\text {tri }}\right)^{-1}$ without a need for computing the whole Green's function and all the eigenvalues/eigenfunctions of the Hamiltonian.

Let us denote $\lambda_{i}=G_{1 i}$. From the definition of the Green's function, we obtain

$$
\left(E-\alpha_{i}\right) \lambda_{i}-\beta_{i-1} \lambda_{i-1}-\beta_{i} \lambda_{i+1}=0, \quad 2 \leqslant i \leqslant N-1
$$

with $\left(E-\alpha_{N}\right) \lambda_{N}-\beta_{N-1} \lambda_{N-1}=0 \quad$ and $\quad\left(E-\alpha_{1}\right) \lambda_{1}-$ $\beta_{1} \lambda_{2}=1$. Expressing sequentially $\lambda_{N}$ by $\lambda_{N-1}, \lambda_{N-1}$ via $\lambda_{N-2}$ and $\lambda_{N-3}$, we express $G_{11}$ as a continued fraction:

$$
G_{11}=\frac{1}{E-\alpha_{1}-\frac{\beta_{1}^{2}}{E-\alpha_{2}-\frac{\beta_{2}^{2}}{\frac{\ddots}{E-\alpha_{M}-\beta_{M}^{2} \Sigma(E)}}}},
$$

In the above equation, we truncated the order- $N$ continued fraction at the fraction $M<N$ by introducing the self-energy $\Sigma(E)$ :

$$
\Sigma(E)=\frac{1}{E-\alpha_{M}-\frac{\beta_{M}^{2}}{E-\alpha_{M}-\frac{\beta_{M}^{2}}{\ddots}}}=\frac{1}{E-\alpha_{M}-\beta_{M}^{2} \Sigma(E)},
$$

which includes all the remaining terms $M+1 \leqslant i \leqslant N$. Solving Eq. (A13), one easily obtains

$$
\Sigma(E)=\frac{E-\alpha_{M}-i \sqrt{4 \beta_{M}^{2}-\left(E-\alpha_{M}\right)^{2}}}{2 \beta_{M}^{2}} .
$$

The number of terms $M$ included in the summation in Eq. (A12) is determined from the condition for the convergence of $G_{11}$. For instance, in our calculations with the $N \times N$ Hamiltonian matrices with $N=1.7 \times 10^{6}$ and $6.8 \times 10^{6}$ it is sufficient to choose $M \approx 2000$ and 4000, respectively.

\section{b. Tridiagonalization of the Hamiltonian matrix}

In order to utilize the continued fraction technique to calculate $G_{11}$, the Hamiltonian should be transformed to the tridiagonalized form, Eq. (A10). This is done by constructing a new orthogonal basis as described below. We start by selecting the first basis vector $\mid 1\}$. We use curled brackets $\mid i\}$ to denote the new basis vectors and straight brackets $|i\rangle$ to denote the old ones. If the tridiagolization is performed in order to find the local density of states on the $i$ th site of the system at hand, the first basis vector is selected as $\mid 1\}=|i\rangle$, where $|i\rangle=c_{i}^{\dagger}|0\rangle$. In most of our calculations, we select the first basis vector as the random state occupying $M$ sites $\mid 1\}=\left|\psi_{\text {ran }}\right\rangle$, with $\left|\psi_{\text {ran }}\right\rangle$ being given by Eq. (A1).

We require that the Hamiltonian in the new basis be of the tridiagonal form (A10). By operating $\hat{H} \mid i\}$, we arrive to the following equations:

$$
\begin{aligned}
\hat{H} \mid 1\} & \left.\left.=\alpha_{1} \mid 1\right\}+\beta_{1} \mid 2\right\}, \\
\hat{H} \mid i\} & \left.\left.\left.=\beta_{i-1} \mid i-1\right\}+\alpha_{i} \mid i\right\}+\beta_{i} \mid i+1\right\}, \quad 2 \leqslant i \leqslant N-2,
\end{aligned}
$$

$\left.\left.\hat{H} \mid N\}=\beta_{N-1} \mid N-1\right\}+\alpha_{N} \mid N\right\}$.

Using Eq. (A15a) and the orthogonality relation $\{1 \mid 2\}=0$, we obtain the second basis vector and the matrix elements $\alpha_{1}$ and $\beta_{1}$ :

$$
\begin{aligned}
\mid 2\} & \left.\left.=\frac{1}{\sqrt{C_{2}}}(\hat{H} \mid 1\}-\alpha_{1} \mid 1\right\}\right), \\
\alpha_{1} & =\{1|\hat{H}| 1\}, \quad \beta_{1}=\{2|\hat{H}| 1\},
\end{aligned}
$$

where the normalization coefficient $C_{2}$ (as well as all other normalization coefficients $C_{i}, 2 \leqslant i \leqslant N$ ) are obtained from the normalization requirement $\{i \mid i\}=1$.

We then proceed to Eq. (A15b) and recursively calculate the basis vectors $\mid i\}, 2 \leqslant i \leqslant N-2$, and corresponding matrix elements $\alpha_{i}$ and $\beta_{i}$ :

$$
\begin{aligned}
\mid i+1\} & \left.\left.\left.=\frac{1}{\sqrt{C_{i+1}}}(\hat{H} \mid i\}-\beta_{i-1} \mid i-1\right\}-\alpha_{i} \mid i\right\}\right), \\
\alpha_{i} & =\{i|\hat{H}| i\}, \quad \beta_{i+1}=\{i+1|\hat{H}| 1\}, \quad 2 \leqslant i \leqslant N-2 .
\end{aligned}
$$

Finally, from Eq. (A15c) we obtain

$$
\left.\left.\mid N\}=\frac{1}{\sqrt{C_{N}}}(\hat{H} \mid N\}-\alpha_{N} \mid N\right\}\right), \alpha_{N}=\{N|\hat{H}| N\}
$$

which concludes the tridiagonalization procedure.

\section{Role of the initial wave-packet size in the averaging over impurity configurations}

For a given configuration of impurities, the conductivity of the system at hand can be sensitive to details of the potential and thus can vary from one impurity realization to another. The conductivity therefore should be averaged by, e.g., performing many calculations for different impurity distributions. Within the present time-dependent Kubo approach, the averaging can be done in a much more efficient way by simply increasing a size of the initial random state without need for many different calculations for different impurity realizations. In this section, we investigate how one can achieve an efficient 

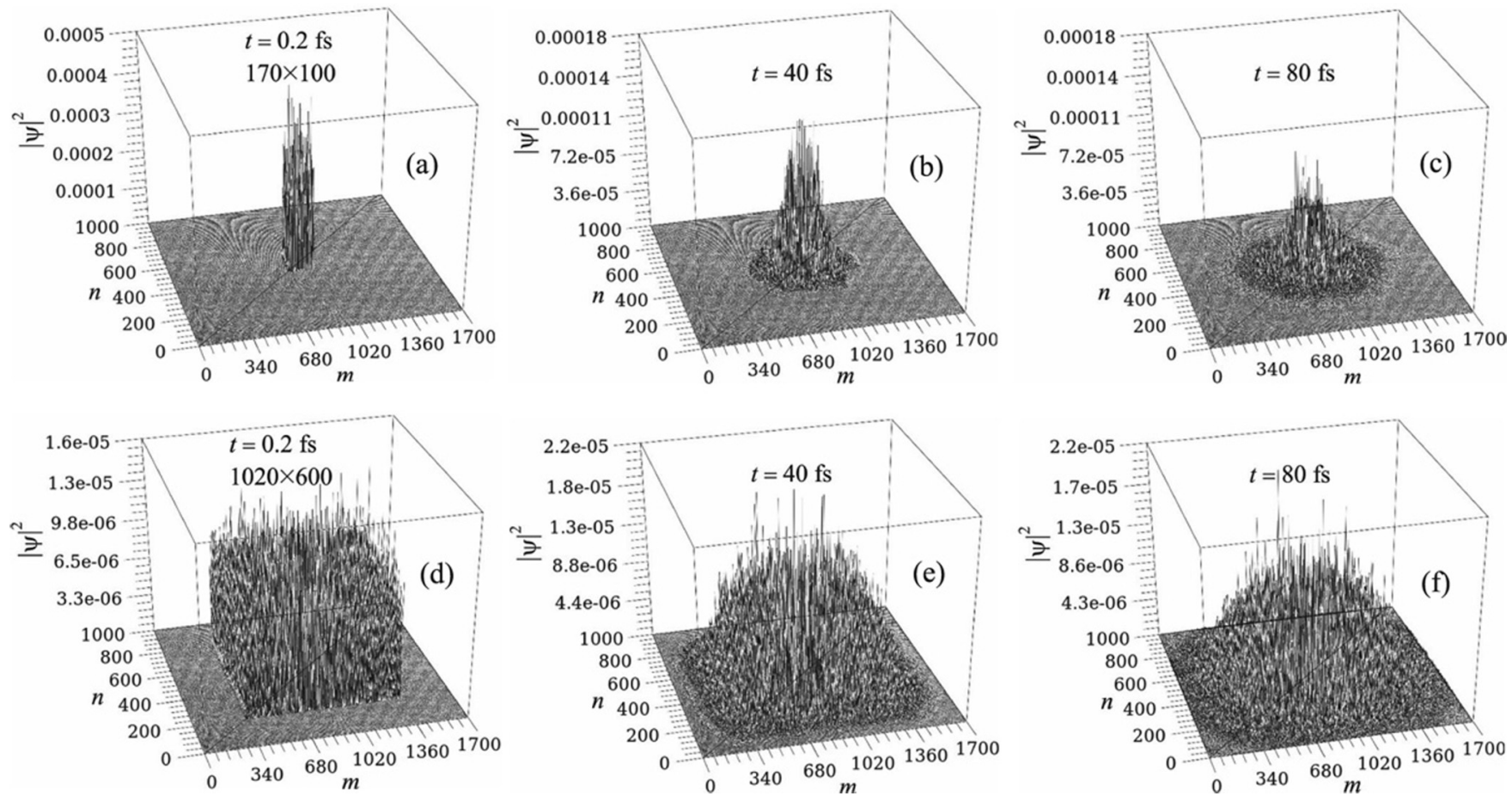

FIG. 8. Propagation of wave packets of two different initial sizes: $170 \times 100$ and $1020 \times 600$ on a graphene sheet with random distribution of the short-range strong impurities $V=37 u, n_{i}=1 \%$.

averaging of the conductivity by varying the size of the wave packet.

Figure 8 shows a temporal evolution of two random initial states $\left|\psi_{\text {ran }}\right\rangle$ [Eq. (A1)] of different sizes: $170 \times 100$ and $1020 \times 600$, respectively. The corresponding time-dependent diffusion coefficients $D(t)$ are shown in Fig. 9 for different electron energies $E$. For the case of the smaller packet, the calculated temporal dependencies show fluctuations caused by the interference within the wave packet. In contrast, for the case of the larger packet, these fluctuations are efficiently averaged out and $D(t)$ exhibit a smooth monotonic behavior for all considered energies. These self-averaging features of the wave packets temporal dynamics manifest themselves in the density dependencies of the conductivity $\sigma=\sigma(n)$. Figures 10(a) and 10(b) show the dependencies $\sigma=\sigma(n)$ for the above wave packets for different impurity realizations. (Note that the concentration of impurities $n_{i}$ in all cases is the same.) For the case of the smaller wave packet, the conductivity exhibits significant fluctuations, whereas for the case of the larger wave packet, the conductivity curves practically coincide. It is important to stress that the averaged values of the conductivity are the same in both cases [see Fig. 10(c)]. Thus, in most of our calculations, we choose the wave packet to be sufficiently large (typically $1020 \times 600$ ) such that no additional averaging over impurity realization is needed.

It is noteworthy that a larger wave packet apparently reaches the boundary of the computational domain earlier than the smaller one [cf. Figs. 8(c) and 8(f)]. The size of the computational domain should be therefore sufficiently large, such that the maximum value of the diffusion coefficient [Eq. (7a)]
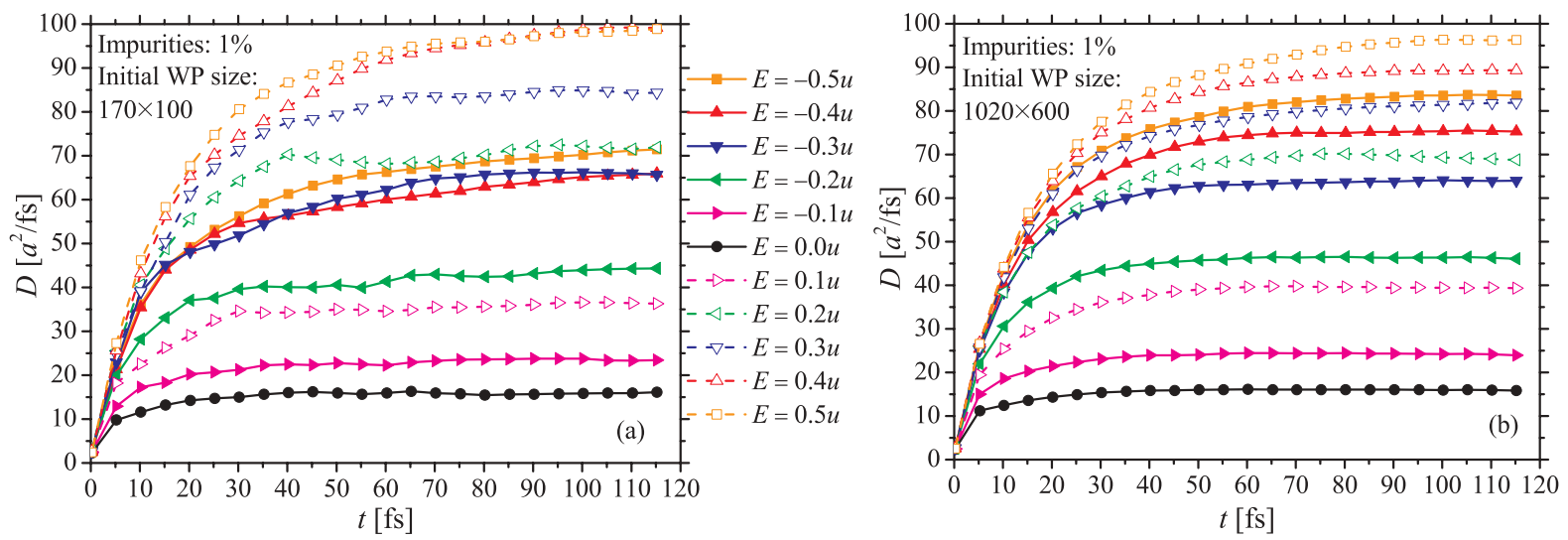

FIG. 9. (Color online) Time-dependent diffusion coefficient $D(t)$ at different energies $E$ for the wave packets (WP) of Fig. 8. 

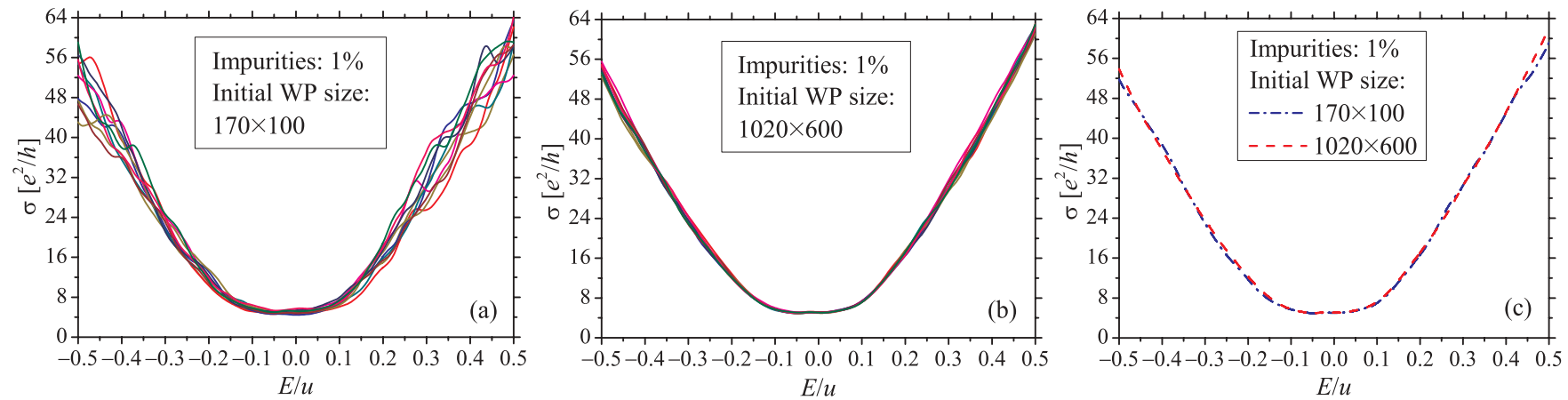

FIG. 10. (Color online) (a), (b) Conductivity vs energy for the wave packet (WP) of Fig. 8 for 10 different impurity configurations. (c) Averaged values of conductivities for the wave packets from (a) and (b).

is reached before the wave packet hits the boundaries. In our calculations, we used the honeycomb lattices of the sizes $1700 \times 1000$ and $3400 \times 2000$ sites (corresponding to $210 \times 210$ and $420 \times 420 \mathrm{~nm}^{2}$, respectively). *igor.zozoulenko@liu.se

${ }^{1}$ A. H. Castro Neto, F. Guinea, N. M. R. Peres, K. S. Novoselov, and

A. K. Geim, Rev. Mod. Phys. 81, 109 (2009).

${ }^{2}$ N. M. R. Peres, Rev. Mod. Phys. 82, 2673 (2010).

${ }^{3}$ S. Das Sarma, S. Adam, E. H. Hwang, and E. Rossi, Rev. Mod. Phys. 83, 407 (2011).

${ }^{4}$ T. Stauber, N. M. R. Peres, and F. Guinea, Phys. Rev. B 76, 205423 (2007).

${ }^{5}$ M. I. Katsnelson and K. S. Novoselov, Solid State Commun. 143, 3 (2007)

${ }^{6}$ P. M. Ostrovsky, I. V. Gornyi, and A. D. Mirlin, Phys. Rev. B 74, 235443 (2006).

${ }^{7}$ J. W. Klos and I. V. Zozoulenko, Phys. Rev. B 82, 081414(R) (2010).

${ }^{8}$ S. Yuan, H. De Raedt, and M. I. Katsnelson, Phys. Rev. B 82, 115448 (2010).

${ }^{9}$ A. Ferreira, J. Viana-Gomes, J. Nilsson, E. R. Mucciolo, N. M. R. Peres, and A. H. Castro Neto, Phys. Rev. B 83, 165402 (2011).

${ }^{10}$ T. Ando, J. Phys. Soc. Jpn. 75, 074716 (2006).

${ }^{11}$ K. Nomura and A. H. MacDonald, Phys. Rev. Lett. 96, 256602 (2006).

${ }^{12}$ E. H. Hwang, S. Adam, and S. Das Sarma, Phys. Rev. Lett. 98, 186806 (2007).

${ }^{13}$ K. S. Novoselov, A. K. Geim, S. V. Morozov, D. Jiang, M. I. Katsnelson, I. V. Grigorieva, S. V. Dubonos, and A. A. Firsov, Nature (London) 438, 197 (2005).

${ }^{14}$ Y.-W. Tan, Y. Zhang, K. Bolotin, Y. Zhao, S. Adam, E. H. Hwang, S. Das Sarma, H. L. Stormer, and P. Kim, Phys. Rev. Lett. 99, 246803 (2007).

${ }^{15}$ S. V. Morozov, K. S. Novoselov, M. I. Katsnelson, F. Schedin, D. C. Elias, J. A. Jaszczak, and A. K. Geim, Phys. Rev. Lett. 100, 016602 (2008).

${ }^{16}$ L. A. Ponomarenko, R. Yang, T. M. Mohiuddin, M. I. Katsnelson, K. S. Novoselov, S. V. Morozov, A. A. Zhukov, F. Schedin, E. W. Hill, and A. K. Geim, Phys. Rev. Lett. 102, 206603 (2009).

${ }^{17}$ M. Monteverde, C. Ojeda-Aristizabal, R. Weil, K. Bennaceur, M. Ferrier, S. Gueron, C. Glattli, H. Bouchiat, J. N. Fuchs, and D. L. Maslov, Phys. Rev. Lett. 104, 126801 (2010).
${ }^{18}$ Z. H. Ni, L. A. Ponomarenko, R. R. Nair, R. Yang, S. Anissimova, I. V. Grigorieva, F. Schedin, Z. X. Shen, E. H. Hill, K. S. Novoselov, and A. K. Geim, Nano Lett. 10, 3868 (2010).

${ }^{19}$ N. J. G. Couto, B. Sacépé, and A. F. Morpurgo, Phys. Rev. Lett. 107, 225501 (2011).

${ }^{20}$ J. P. Robinson, H. Schomerus, L. Oroszlany, and V. I. Fal'ko, Phys. Rev. Lett. 101, 196803 (2008).

${ }^{21}$ T. O. Wehling, S. Yuan, A. I. Lichtenstein, A. K. Geim, and M. I. Katsnelson, Phys. Rev. Lett. 105, 056802 (2010).

${ }^{22}$ N. Leconte, A. Lherbier, F. Varchon, P. Ordejon, S. Roche, and J.-C. Charlier, Phys. Rev. B 84, 235420 (2011).

${ }^{23}$ A. Lherbier, S. M.-M. Dubois, X. Declerck, Y.-M. Niquet, S. Roche, and J.-Ch. Charlier, arXiv:1204.4574.

${ }^{24}$ G. Trambly, de Laissardiere, and D. Mayou, Mod. Phys. Lett. B 25, 1019 (2011).

${ }^{25}$ B. Huard, N. Stander, J. A. Sulpizio, and D. Goldhaber-Gordon, Phys. Rev. B 78, 121402(R) (2008).

${ }^{26}$ S. Barraza-Lopez, M. Vanevic, M. Kindermann, and M. Y. Chou, Phys. Rev. Lett. 104, 076807 (2010).

${ }^{27}$ Bo-Chao Huang, Ming Zhang, Yanjie Wang, and Jason Woo, Appl. Phys. Lett. 99, 032107 (2011).

${ }^{28}$ X. Du, I. Skachko, A. Barker, and E. Y. Andrei, Nat. Nanotechnol. 3, 491 (2008).

${ }^{29}$ K. I. Bolotin, K. J. Sikes, J. Hone, H. L. Stormer, and P. Kim, Phys. Rev. Lett. 101, 096802 (2008).

${ }^{30}$ J. W. Klos, A. A. Shylau, I. V. Zozoulenko, Hengyi Xu, and T. Heinzel, Phys. Rev. B 80, 245432 (2009).

${ }^{31}$ E.-A. Kim and A. H. Castro Neto, Europhys. Lett. 84, 57007 (2008).

${ }^{32}$ M. I. Katsnelson and A. K. Geim, Philos. Trans. R. Soc. London A 366, 195 (2008).

${ }^{33}$ C. H. Lewenkopf, E. R. Mucciolo, and A. H. Castro Neto, Phys. Rev. B 77, 081410(R) (2008).

${ }^{34}$ H. Xu, T. Heinzel, M. Evaldsson, and I. V. Zozoulenko, Phys. Rev. B 77, 245401 (2008).

${ }^{35}$ S. Ihnatsenka and G. Kirczenow, Phys. Rev. B 83, 245442 (2011). 
${ }^{36}$ S. Roche and D. Mayou, Phys. Rev. Lett. 79, 2518 (1997).

${ }^{37}$ F. Triozon, J. Vidal, R. Mosseri, and D. Mayou, Phys. Rev. B 65, 220202(R) (2002).

${ }^{38}$ F. Triozon, S. Roche, A. Rubio, and D. Mayou, Phys. Rev. B 69, 121410(R) (2004).

${ }^{39}$ T. Markussen, R. Rurali, M. Brandbyge, and A.-P. Jauho, Phys. Rev. B 74, 245313 (2006); T. Markussen, Master's thesis, Technical University of Denmark, 2006.

${ }^{40}$ H. Ishii, N. Kobayashi, and K. Hirose, Phys. Rev. B 82, 085435 (2010).

${ }^{41}$ Jun Yan and M. S. Fuhrer, Phys. Rev. Lett. 107, 206601 (2011).

${ }^{42}$ Qiuzi Li, E. H. Hwang, E. Rossi, and S. Das Sarma, Phys. Rev. Lett. 107, 156601 (2011).

${ }^{43}$ Hengyi Xu, T. Heinzel, and I. V. Zozoulenko, Phys. Rev. B 84, 115409 (2011).

${ }^{44} \mathrm{~A}$ graphene lattice with an adatom situated at the $j$ th site can be described by the Hamiltonian $\hat{H}=-u \sum_{i, i^{\prime}} c_{i}^{\dagger} c_{i^{\prime}}-u_{a d}\left(c_{j}^{\dagger} a_{j}+\right.$ $\left.c_{j}^{\dagger} a_{j}\right)+\epsilon_{a d} c_{j}^{\dagger} c_{j}$, where the operators $c_{j}$ and $a_{j}$ correspond to, respectively, graphene lattice and the adatom, $u$ is defined in Eq. (1), $u_{a d}$ is the hopping integral between the adatom and the carbon atom of the graphene lattice at the site $j$, and $\epsilon_{a d}$ is the onsite energy at the adatom. Using the $T$-matrix theory, one can show that this Hamiltonian can be reduced to Eq. (1) where the strength of the onsite potential in Eq. (2) is (Refs. 9,20,21, and 35) $V_{0}=u_{a d}^{2} /\left(E-\epsilon_{a d}\right)$. With typical values of $u_{a d}$ and $\epsilon_{a d}$ provided by the ab initio calculations, the effective onsite potentials in the vicinity of the Dirac point in the range $V_{0} / u \approx 10-80$ (Ref. 9).

${ }^{45}$ S. Adam, P. W. Brouwer, and S. Das Sarma, Phys. Rev. B 79, 201404(R) (2009).

${ }^{46} \mathrm{O}$. Madelung, Introduction to Solid-State Theory (Springer, Berlin, 1996).

${ }^{47}$ Note that the general definition of the diffusion coefficient is $D_{x}(E, t)=\frac{d\left\langle\Delta \hat{X}^{2}(E, t)\right\rangle}{d t}$. This definition becomes equivalent to Eq. (7a) in a classical diffusive regime when $D$ is independent of time and the mean spreading of a wave packet is linearly proportional to time, $\left\langle\Delta \hat{X}^{2}(E, t)\right\rangle \propto t$. However, even in the case when $D$ depends on $t$, both definitions lead to qualitatively the same results (Ref. 23). This justifies utilization of Eq. (7a), which is less demanding computationally.

${ }^{48}$ In our numerical calculations, we use relative electron densities in the range $n_{e} \lesssim 0.06\left(n \lesssim 2.3 \times 10^{14} \mathrm{~cm}^{-2}\right)$. This is larger than those typically used in experiments, $n_{e}^{\exp } \lesssim 5 \times 10^{-5}\left(n^{\exp } \lesssim 2 \times\right.$ $\left.10^{11} \mathrm{~cm}^{-2}\right)$. We use larger electron density interval because we model conductivity using impurity densities $n_{i} \lesssim 5 \%\left(n_{i} \lesssim 2 \times\right.$ $\left.10^{11} \mathrm{~cm}^{-2}\right)$, which are larger than those in typical experimental samples $n_{i}^{\exp } \lesssim 0.05 \%\left(n_{i}^{\exp } \approx 2 \times 10^{11} \mathrm{~cm}^{-2}\right) .^{2}$ This is due to the fact that with the realistic impurity densities, one would need to perform calculations on graphene sheets with thousands of millions of atoms in order to achieve the diffusive transport regime, which exceeds the present computation capabilities.

${ }^{49}$ F. T. Vasko and V. Ryzhii, Phys. Rev. B 76, 233404 (2007). 